\title{
Lung inflammatory pattern and antibiotic treatment in pneumonia
}

\author{
María-José Lorenzo ${ }^{1,4,5}$, Inés Moret² ${ }^{2}$ Benjamín Sarria ${ }^{3}$, Enrique Cases ${ }^{1}$, Julio Cortijo ${ }^{3,7}$, Raúl Méndez ${ }^{1}$, Jose Molina ${ }^{6}$, \\ Alejandra Gimeno ${ }^{1}$ and Rosario Menéndez ${ }^{1,7,8^{*}}$
}

\begin{abstract}
Background: In community-acquired pneumonia host inflammatory response against the causative microorganism is necessary for infection resolution. However an excessive response can have deleterious effects. In addition to antimicrobial effects, macrolide antibiotics are known to possess immunomodulatory properties.

We aimed to evaluate inflammatory cytokine profiles - both locally (bronchoalveolar lavage) and systemically (blood) - in community-acquired pneumonia admitted patients after at least 72 hours of antibiotic treatment (with and without macrolide containing regimens) and requiring bronchoscopic examination for inadequate response due to infection progression and/or lack of clinical stability.

Methods: A prospective study was performed on 52 admitted patients who developed an inadequate response after 72 hours of antibiotic treatment - non-responders community-acquired pneumonia - (blood and bronchoalveolar lavage), and two control groups: 1) community-acquired pneumonia control (blood) and 2) non-infection control (blood and bronchoalveolar lavage). Cytokine profiles (interleukin (IL)-6, IL-8, IL-10), tumour necrosis factor a and clinical outcomes were assessed.
\end{abstract}

Results: Non-responders patients treated with macrolide containing regimens showed significantly lower levels of IL-6 and TNF-a in bronchoalveolar lavage fluid and lower IL-8 and IL-10 in blood than those patients treated with nonmacrolide regimens. Clinical outcomes showed that patients treated with macrolide regimens required fewer days to reach clinical stability $(p<0.01)$ and shorter hospitalization periods $(p<0.01)$.

Conclusions: After 72 hours of antibiotic effect, patients who received macrolide containing regimens exhibited lower inflammatory cytokine levels in pulmonary and systemic compartments along with faster stabilization of infectious parameters.

Keywords: Community acquired pneumonia, Macrolides, Lung inflammation

\section{Introduction}

The majority of hospitalized community-acquired pneumonia (CAP) patients respond favorably to antibiotic treatment but around $10 \%$ develop an inadequate response to treatment that leads to a poorer prognosis [1]. Cytokines are important mediators that orchestrate the inflammatory response and hence play an important role in host defense against microorganisms. However, excessive and persistent cytokine production [2] has been associated with a higher number of days needed to reach

\footnotetext{
* Correspondence: rosmenend@gmail.com

${ }^{1}$ Pneumology Department, Instituto de Investigación Sanitaria del Hospital Universitario y Politécnico La Fe (IIS La Fe), Valencia, Spain

${ }^{7}$ CIBER de Enfermedades Respiratorias (CibeRes), Valencia, Spain

Full list of author information is available at the end of the article
}

clinical stability, treatment failure and increased mortality $[3,4]$. After antibiotic treatment, when the response is adequate, inflammation decreases with a return to homeostasis.

The immunomodulatory effects of macrolides benefit the host due to their capacity to temper the production of inflammatory cytokines [5-7]. Some recent observational studies have reported better outcomes in severe CAP cases [8-12], when antibiotics regimens contained macrolides. Others studies, though, have not reported such improvement $[13,14]$. We hypothesized that antibiotic influence on cytokine production might have a beneficiary effect upon the resolution of infectious parameters. Although systemic inflammation has been examined 
previously, research into lung cytokine patterns in CAP with different antibiotics regimens is scarce.

The aims of the present study were to investigate 1) lung and systemic inflammatory cytokine profiles in hospitalized CAP patients after at least 72 hours of antibiotic treatment (with and without macrolide containing regimens) and who required bronchoscopic examination owing to an inadequate response consequent upon infection progression and/or lack of clinical stability; and 2) the impact of macrolide containing regimens treatment on clinical resolution parameters.

\section{Methods}

\section{Study design}

A prospective longitudinal study was conducted of admitted CAP patients requiring bronchoscopic examination due to an inadequate response to antibiotic treatment after at least 72 hours of antibiotic treatment: non-responders CAP (NCAP). The inclusion criteria for the NCAP study group were: 1) clinical deterioration with acute respiratory failure requiring ventilator support and/or septic shock; and/or 2) persistence of a high temperature $\left(\geq 38^{\circ} \mathrm{C}\right)$ and/or clinical symptoms, and/or chest- $\mathrm{X}$ ray progression $(>50 \%$ increase of infiltrates with clinical symptoms) or empyema [3]. Patient enrolment in the study was performed on the day of their bronchoscopic examination when samples of bronchoalveolar (BAL) fluid and blood were obtained.

Two control groups were included: 1) CAP control group (CAP), comprised of patients of a similar age and co-morbid condition who reached clinical stability after 72 hours of antibiotic treatment (temperature $<37.2^{\circ} \mathrm{C}$, heart rate $<100$ beats/min, respiratory rate $<24$ breaths/ min, systolic blood pressure $>90 \mathrm{~mm} \mathrm{Hg}$, and oxygen saturation $>90 \%$ or arterial oxygen tension $>60 \mathrm{~mm} \mathrm{Hg}$ when patient was not receiving supplemental oxygen) $[15,16]$; and 2) Non-infection control group, comprised of patients without any infection for whom a bronchoscopy had been scheduled due to peripheral lung nodules or minor haemoptysis.

Exclusion criteria were: prior admission to hospital (1 month), immunosuppressive treatment, HIV infection, or alternative diagnosis.

The study was approved by the Ethical Committee of our hospital (approval number 2005/0141) and patients signed the required informed consent form.

\section{Data collection}

The following data were collected: demographics, comorbid conditions, laboratory data, chest radiograph, initial pneumonia severity [17], microbiological tests (sputum culture, urinary antigens of Legionella pneumophila and Streptococcus pneumoniae, blood cultures and serology for Chlamydophila pneumoniae, Mycoplasma pneumonia,
Coxiella burnetii and Legionella pneumophila), and length of hospital stay (LOS) which was calculated as the number of days from admission until discharge.

Antimicrobial therapy received by patients was recorded and classified as follows: beta-lactams (ceftriaxone/cefotaxime: 1-2 g/12-24 h, 1-2 g/8 h or co-amoxiclavulanate: 1-2 g/8 h) + macrolides (azithromycin: $500 \mathrm{mg} / 24 \mathrm{~h}$ ); fluoroquinolone (levofloxacin: $500 \mathrm{mg} / 12-24 \mathrm{~h}$ ) in monotherapy, both as recommended by Spanish guidelines [18], and beta-lactams + fluoroquinolone. Patients who received any other combination of antibiotics were classified as "other regimens".

\section{Biological samples collection}

In both the NCAP and non-infection control group, BAL fluid and blood samples were obtained on the day of bronchoscopic examination. In the CAP control group, blood samples were obtained at clinical stability, after at least 72 hours of antibiotic treatment, and no BAL fluid was obtained.

\section{Bronchoalveolar lavage}

BAL was collected according to recommended guidelines [19] by flexible videobronchoscope in the affected lobe in NCAP patients and in the middle lobe in noninfection controls. Five aliquots of sterile saline solution were instilled and immediately aspirated. The first aliquot $(20 \mathrm{~mL})$ was discarded. The remaining four aliquots $(30 \mathrm{~mL}$ each) were pooled together in a single sterile glass: $50 \%$ of the retrieved fluid was sent to the microbiology laboratory and the other $50 \%$ to the biochemistry laboratory for cytokine measurement. The mean \pm SEM BAL fluid obtained for processing was $56 \pm 2 \mathrm{ml}$. To eliminate remaining mucus, the samples were filtered through two sheets of gauze. BAL fluid sample was centrifuged at $353 \times \mathrm{g}$ for $5 \mathrm{~min}$ at $4^{\circ} \mathrm{C}$. The supernatant volume was measured and frozen at $-80^{\circ} \mathrm{C}$ until further analysis.

\section{Blood samples}

Venous blood samples were collected prior to the BAL procedure and were centrifuged at $1500 \mathrm{rpm}$ for 10 minutes. Plasma was separated, coded and frozen at $-80^{\circ} \mathrm{C}$ until further processing.

\section{Cytokines determinations}

The determination of cytokines levels [interleukin (IL)-6, IL-8, IL-10 and tumour necrosis factor $\alpha$ (TNF- $\alpha)$ ] were analysed using the commercially available enzyme immunoassay (Pharmingen, BD Biociencias, Madrid, Spain) according to the manufacturer's protocol. The limits of detection were $3 \mathrm{pg} / \mathrm{ml}$ for IL-6, $3 \mathrm{pg} / \mathrm{ml}$ for IL-8, $2 \mathrm{pg} / \mathrm{ml}$ for IL-10 and $1 \mathrm{pg} / \mathrm{ml}$ for TNF- $\alpha$. 


\section{Statistical analysis}

Statistical analysis was performed with the SPSS statistical software package, version 12.0 (SPSS, Chicago, IL, USA), and the GraphPad software (San Diego, CA, USA). Chi-square and Mann-Whitney U tests were performed for, respectively, the qualitative and quantitative variables. Data are shown as mean \pm SEM. Statistical significance was considered at a $\mathrm{p}$ value of $<0.05$.

\section{Results}

\section{Patient characteristics}

86 patients were included: 52 NCAP, 15 CAP controls and 19 non-infection controls. The main demographic characteristics, comorbidity, initial pneumonia severity and antimicrobial therapy for each group are shown in Table 1. The median days of hospitalization prior to enrolment in the study was 5 days in the NCAP group and 4 in the CAP control group. The reasons for nonresponse in the NCAP group were: 32 (61.5\%) persistent

Table 1 General characteristics, comorbidity, initial pneumonia severity and antimicrobial therapy of the study population

\begin{tabular}{|c|c|c|c|c|}
\hline & $N C A P$ & $\begin{array}{l}\text { CAP } \\
\text { control }\end{array}$ & $\begin{array}{l}\text { Non-infection } \\
\text { control }\end{array}$ & $p$ value $^{*}$ \\
\hline Subjects, n & 52 & 15 & 19 & \\
\hline Age, years & $61 \pm 2$ & $66 \pm 4$ & $61 \pm 2$ & NS \\
\hline Male/Female & $31 / 21$ & $12 / 3$ & $16 / 3$ & NS \\
\hline Current smoker, n & 16 & 2 & 9 & NS \\
\hline \multicolumn{5}{|l|}{ Comorbidity, n } \\
\hline - Cerebrovascular disease & $11(21)$ & $3(20)$ & $2(10)$ & NS \\
\hline - Heart disease & $12(23)$ & $3(20)$ & $2(10)$ & NS \\
\hline - COPD & $7(14)$ & $7(46)$ & $7(37)$ & 0.01 \\
\hline PSI score & $98 \pm 4$ & $102 \pm 7$ & $\mathrm{~N} / \mathrm{A}$ & NS \\
\hline CRP (mg/dl) & & & $\mathrm{N} / \mathrm{A}$ & \\
\hline - At admission & $232 \pm 25$ & $190 \pm 30$ & & NS \\
\hline - At enrolment & $165 \pm 18$ & $68 \pm 21$ & & 0.05 \\
\hline Inhaled corticosteroids & $2(29)$ & $1(14)$ & $0(15)$ & NS \\
\hline Systemic corticosteroids & $2(29)$ & $3(43)$ & 0 & NS \\
\hline Antimicrobial therapy ${ }^{\beta}, \mathrm{n}$ & & & N/A & \\
\hline - Fluoroquinolone & $6(12)$ & $5(33)$ & & \\
\hline - $\beta$-lactam plus macrolide & $23(44)$ & $9(60)$ & & \\
\hline $\begin{array}{l}\text { - } \beta \text {-lactam plus } \\
\text { fluoroquinolone }\end{array}$ & $8(15)$ & 0 & & \\
\hline - Others regimens ${ }^{* *}$ & $15(28)$ & $1(7)$ & & \\
\hline
\end{tabular}

Data are expressed as mean \pm SEM or $n(\%)$. *Mann-Whitney $U$ test and chi-square test for continuous and categorical variables, respectively.

NCAP: non-responders pneumonia; CAP: community-acquired pneumonia; COPD: chronic obstructive pulmonary disease; PSI score: Pneumonia Severity Index score; CRP: C-reactive protein; NS: non-significant. N/A: not applicable.

${ }^{\beta}$ Antimicrobial therapy at enrolment of the study.

Other regimens ${ }^{* *}$ vancomycin, imipemen-cilastatin, piperacillin-tazobactam, amikacin, clindamycin, ceftazidime, ertapenem and linezolid. fever and clinical worsening; $15(28.8 \%)$ radiological progression- 2 of these with pleural effusion-; and 5 (9.6\%) respiratory failure requiring invasive ventilation. Six patients died during hospitalization.

General characteristics, comorbidity conditions and corticosteroids treatment according to macrolides regimens in the NCAP and CAP control groups are shown in Table 2. The median macrolide dosages for patients at the point of enrolment for the study were: 5 dosages of azithromycin $500 \mathrm{mg} / 24 \mathrm{~h}$ iv for the NCAP group and 3 dosages of azithromycin $500 \mathrm{mg} / 24 \mathrm{~h}$ iv for the CAP control group.

\section{Microbiological results}

In the NCAP group, etiological microorganism was identified in 24 patients (46\%). The most frequent isolated pathogen was Streptococcus pneumoniae (13 patients 25\%): 3 in both blood culture and urinary antigen, 7 urinary antigen, 1 blood culture and 2 in BAL. The aetiological diagnosis with regard to macrolide regimens or otherwise, is provided in Table 3. In the CAP control group, aetiological diagnosis was reached in 5 patients (33.3\%): 4 Streptococcus pneumoniae, 1 Pseudomonas aeruginosa.

\section{Cytokine levels in BAL and blood samples NCAP group}

The comparison between patients treated with and without macrolide regimens showed: In BAL fluid, IL-6 and TNF- $\alpha$ were significantly lower in patients treated with macrolide regimens $(216 \pm 66$ vs $590 \pm 230 \mathrm{pg} / \mathrm{mL} ; \mathrm{p}=0.01$ and $1 \pm 0.3$ vs $4 \pm 0.8 ; p=0.03$ respectively), (Figure 1 ), with a trend towards lower IL-8 levels $(p=0.06)$. After excluding patients with inhaled or systemic concomitant corticosteroids treatment, lower IL-6 levels in BAL $(111 \pm 32$ vs $706 \pm 300$; $\mathrm{p}=0.004$ ) were confirmed.

In blood, IL-8 and IL-10 were significantly lower in patients with macrolide regimens $(42 \pm 13$ vs $57 \pm 13$; $\mathrm{p}=0.04$, and $15 \pm 6$ vs $27 \pm 7 ; \mathrm{p}=0.01$ respectively), (Figure 2), with a trend towards lower IL-6 levels $(\mathrm{p}=0.06)$. No detectable levels of TNF- $\alpha$ in blood were obtained. In the subset of patients without corticosteroids concomitant treatment we corroborated lower IL-6, IL-8 and IL-10 levels in patients with macrolide regimens $(96 \pm 39$ vs $305 \pm 137, \mathrm{p}=0.05 ; 35 \pm 14$ vs $52 \pm 14, \mathrm{p}=0.05$; and $14 \pm 6$ vs $23 \pm 6, \mathrm{p}=0.02$, respectively).

\section{CAP control group}

No significant differences in blood cytokine levels were observed between patients treated with macrolide containing regimens and those treated with non-macrolide regimens (Figure 2) in the whole group and after excluding patients with corticosteroid concomitant treatment. Similarly, no differences were observed between COPD and non-COPD patients. 
Table 2 General characteristics, comorbidity and corticosteroids treatment in NCAP and CAP control groups according to macrolide containing regimens and non-macrolide regimens

\begin{tabular}{|c|c|c|c|c|c|c|}
\hline & \multicolumn{3}{|l|}{ NCAP } & \multicolumn{3}{|c|}{ CAP controls } \\
\hline & $\begin{array}{l}\text { Macrolide } \\
\text { regimens }^{a}\end{array}$ & $\begin{array}{l}\text { Non-macrolide } \\
\text { regimen }^{\beta}\end{array}$ & $p$ value* & $\begin{array}{l}\text { Macrolide } \\
\text { regimens }^{a}\end{array}$ & $\begin{array}{l}\text { Non-macrolide } \\
\text { regimens }^{\beta}\end{array}$ & $p$ value* \\
\hline Subjects, $n$ & 23 & 29 & & 9 & 6 & \\
\hline Age, years & $60 \pm 4$ & $63 \pm 3$ & NS & $78 \pm 3$ & $70 \pm 9$ & NS \\
\hline Male/Female & $12 / 11$ & $19 / 10$ & NS & $7 / 2$ & $5 / 1$ & NS \\
\hline \multicolumn{7}{|l|}{ Co-morbidity, n } \\
\hline - Cerebrovascular disease & $4(17)$ & $7(24)$ & NS & $3(33)$ & 0 & NS \\
\hline - Heart disease & $4(17)$ & $8(28)$ & NS & $2(22)$ & $1(17)$ & NS \\
\hline - COPD & $1(4)$ & $6(21)$ & NS & $3(33)$ & $4(67)$ & NS \\
\hline Risk class of Fine & & & NS & & & NS \\
\hline$|-| \mid$ & $7(30)$ & $6(21)$ & & 0 & $1(17)$ & \\
\hline III-V & $16(70)$ & $23(79)$ & & $9(100)$ & $5(83)$ & \\
\hline Inhaled corticosteroid & 0 & $2(7)$ & NS & $1(11)$ & 0 & NS \\
\hline Systemic corticosteroid & $3(13)$ & $6(21)$ & NS & $4(44)$ & $2(33)$ & NS \\
\hline
\end{tabular}

Data are expressed as mean \pm SEM or $\mathrm{n}(\%)$. *Mann-Whitney $U$ test and chi-square test for continuous and categorical variables, respectively.

${ }^{a}$ Macrolide regimens: beta-lactam (ceftriaxone, cefotaxime or co-amoxi-clavulanate + macrolide (azithromicyn: $500 \mathrm{mg} / 24 \mathrm{~h}$ ).

${ }^{\beta}$ Non-macrolide regimens: fluoroquinolone (levofloxacin: $500 \mathrm{mg} / 12-24 \mathrm{~h}$ ) in monotherapy or beta-lactam + fluoroquinolone or other regimens.

NCAP: non-responders pneumonia; CAP: community-acquired pneumonia; COPD: chronic obstructive pulmonary disease; NS: non-significant. N/A: not applicable.

\section{Clinical outcomes}

The relationship between the days needed to reach clinical stability and LOS according to treatment with macrolide and non-macrolide regimens in the NCAP group and the CAP control group is shown in Table 4.

In the NCAP group, patients with macrolide containing regimens required fewer days than patients with non-macrolide regimens to reach clinical stability, and had shorter LOS. After excluding patients with corticosteroid concomitant treatment we confirmed significantly shorter LOS in patients treated with macrolide regimens $(13 \pm 1.5$ vs $24 \pm 4, \mathrm{p}=0.01$, respectively) and fewer days to reach clinical stability $(8 \pm 1$ vs $16 \pm 3, p=$ 0.004 , respectively). No differences were found in the whole CAP control group and after excluding those with corticosteroid treatments.

\section{Discussion}

The most important findings of our study are: 1) Levels of IL- 6 and TNF- $\alpha$ in BAL and of IL- 8 and IL-10 in blood were significantly lower in NCAP patients who received macrolide containing regimens than in those treated with non-macrolide regimens; and 2) there were improved clinical outcomes, such as earlier clinical stability and shorter LOS, in patients who received macrolide containing regimens. Our main findings were also confirmed in patients without any concomitant corticosteroid treatment.

Table 3 Aetiological diagnosis in NCAP and CAP control group patients according to macrolide containing regimens and non-macrolide regimens

\begin{tabular}{|c|c|c|c|c|}
\hline & \multicolumn{2}{|l|}{ NCAP } & \multicolumn{2}{|l|}{ CAP control } \\
\hline & $\begin{array}{l}\text { Macrolide regimens } \\
\text { (n 23) }\end{array}$ & $\begin{array}{l}\text { Non-macrolide regimens } \\
\text { (n 29) }\end{array}$ & $\begin{array}{l}\text { Macrolide regimens } \\
\text { (n 9) }\end{array}$ & $\begin{array}{l}\text { Non-macrolideregimens } \\
\text { (n 6) }\end{array}$ \\
\hline \multicolumn{5}{|l|}{ Microorganisms } \\
\hline - Streptococcus pneumoniae & $5(21.7)$ & $8(27.6)$ & $2(22.2)$ & $2(33.3)$ \\
\hline - Pseudomonas aeruginosa & $2(8.7)$ & $5(17.2)$ & $1(11.1)$ & 0 \\
\hline - Escherichia coli & 0 & $2(6.9)$ & 0 & 0 \\
\hline - Streptococcus spp & $1(4.3)$ & $1(3.4)$ & 0 & 0 \\
\hline - MRSA & 0 & $2(6.9)$ & 0 & 0 \\
\hline - Polymicrobial & 0 & $7(24.1)$ & 0 & 0 \\
\hline Pathogens isolated & $8(34.8)$ & $16(55.2)$ & $3(33.3)$ & 2(33.3) \\
\hline
\end{tabular}

Date are presented as $\mathrm{n}(\%)$.

NCAP: non-responders pneumonia; CAP: community-acquired pneumonia; MRSA: methilcilin-resistant Staphylococcus aureus. 


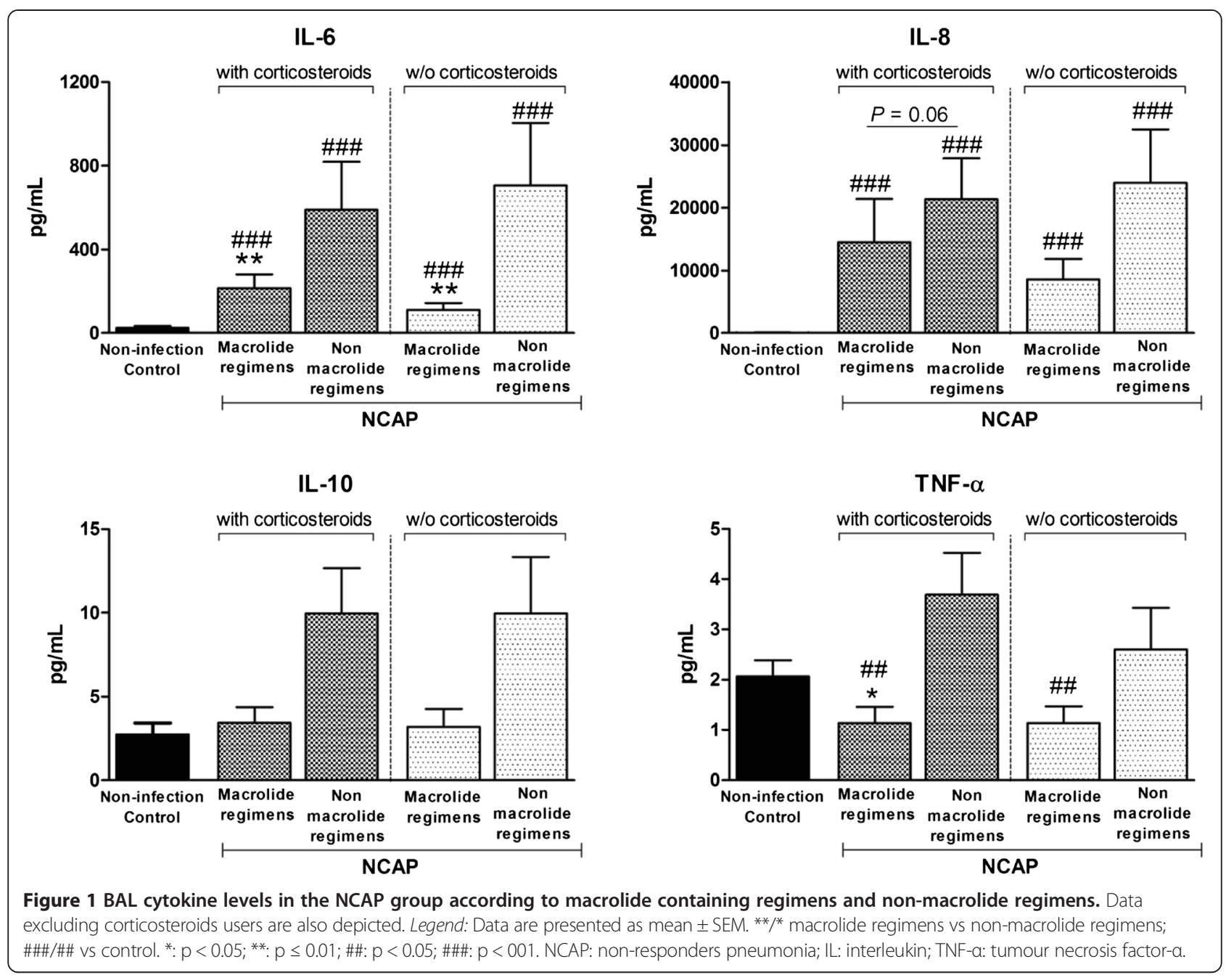

In this study, patients who did not reach clinical stability at 72 hours after antibiotic treatment developed raised cytokine inflammatory levels in both compartments, pulmonary and systemic, thereby following a pattern similar to that found in previous reports. Persistently high levels of IL-6, IL-8 and IL-10 in BAL fluid or serum have been found in severe pneumonia and NCAP $[2,4,20,21]$, reflecting an ongoing inflammation. In this context, declining levels of biomarkers, IL-6, IL-8 and IL-10, along with apoptosis of neutrophils have also been reported at clinical stability [22].

The beneficial impact of controlling inflammatory response through immunodulatory agents in CAP has received significant attention [5,23-26]. In our study, NCAP patients treated with macrolide containing regimens exhibited a trend towards IL-8 BAL fluid levels and significantly lower IL- 6 and TNF- $\alpha$ compared to patients treated with non-macrolide regimens. Moreover, in blood our results also confirmed lower levels of IL-6, IL-8 and IL-10 in the NCAP patients treated with macrolide regimens, while in those CAP control patients who attained clinical stability no significant differences were detected.

Despite the recognized role of macrolides in tempering inflammation, there are very few studies on humans of this [25]. In cases of CAP, Demartini et al. [27] compared systemic cytokines levels of clarithromycin (500 mg twice a day for 7 days) and amoxicylin ( $1 \mathrm{~g}$ three times a day for 7 days) in patients before starting antibiotic therapy, at the 3rd and 7th days of therapy. Clarithromycin decreased significantly levels of IL-6 and increased levels of IL-10 at the 3rd and 7th days in comparison with basal levels. In a randomized clinical study of ventilator associated pneumonia patients, Spyridaki et al. [28] analyzed the effect of the clarithromycin and placebo on cytokines over six consecutive days. The serum ratio of IL-10 to TNF- $\alpha$ decreased in the clarithromycin group compared to the placebo group.

It is also worth highlighting that in our study the effect of macrolides was not apparent in CAP patients with clinical stability, which indicates that, at that point, cytokine levels were already reduced. 


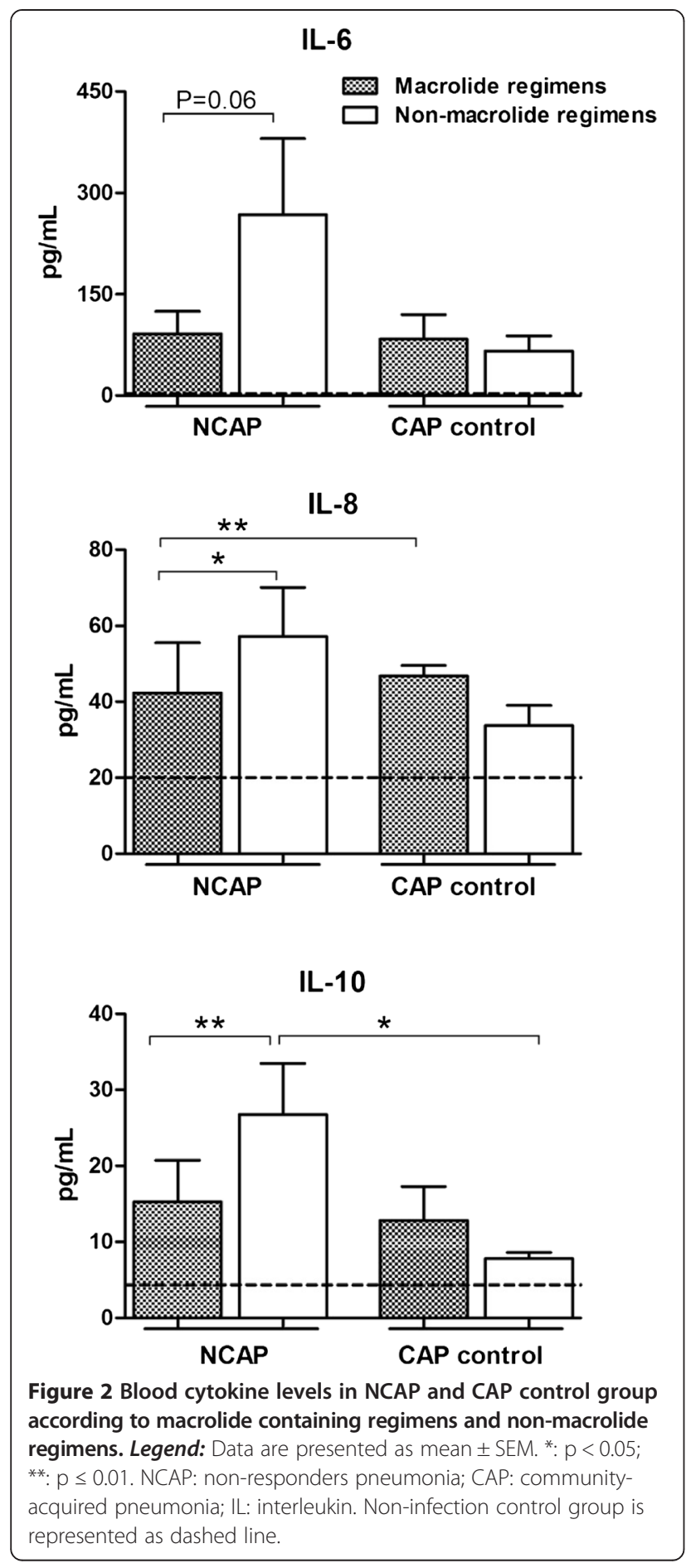

Macrolides inhibit intracellular signaling pathways, suppress the production of NF-kB and the synthesis and/or secretion of pro-inflammatory cytokines, and decrease influx and neutrophil activity [23]. In animal models with pneumonia, the reduction of chemokine secretion and cytokine levels was demonstrated when microorganisms were resistant to macrolides, suggesting that benefits were
Table 4 Comparison of clinical outcomes in NCAP and CAP control group according to macrolide containing regimens and non-macrolide regimens

\begin{tabular}{lllll}
\hline & $\begin{array}{l}\text { Clinical } \\
\text { stability } \\
\text { (days) }\end{array}$ & p\# & LOS & p\# \\
\hline NCAP & & & & \\
-Macrolide regimens (n 23) & $8(6-12)$ & 0.007 & $12(10-21)$ & 0.007 \\
-Non-macrolide regimens ( $n$ 29) & $14(8-27)$ & & $20(13-36)$ & \\
CAP control & & & & \\
-Macrolide regimens $(n$ 9) & $6(3-10)$ & 0.6 & $9(7-16)$ & 0.8 \\
-Non-macrolide regimens $(n$ 6) & $6(3-6)$ & & $9(8-12)$ & \\
\hline Data are presented as median and interguatile range. $p \#$ values were &
\end{tabular}

Data are presented as median and interquartile range. $p \#$ values were calculated using the Mann-Whitney U-test.

NCAP: non-responders pneumonia; CAP: community-acquired pneumonia; LOS: Length of stay.

independent of antimicrobial activity [29]. Sanz et al. [30], reported in respect of an animal study in vivo that erythromycin exerts anti-inflammatory activity and inhibits leukocyte recruitment in the lung. In in vitro studies, results suggest that macrolides exhibit a suppressive effect on cytokines in models of acute inflammation [25], viral bronchiolitis [31] and ventilator-induced lung injury [32]. Anderson et al. [33], have reported that the production of pneumolysin, a key virulence factor of the pneumococcus, is attenuated by exposure of this microbial pathogen to clarithromycin.

The beneficial impact of macrolides on outcomes in CAP cases has been recently evaluated in a systematic review [23] that identified 6 uncontrolled studies: 4 in favor of macrolides [12,34-36] and 2 without beneficial effects $[13,14]$. Moreover, in severe sepsis due to CAP, Restrepo et al. [10], found that patients who received macrolide therapy in comparison with those patients who did not receive it were associated with decreased mortality at 30 days $(11 \%$ vs $29 \%, \mathrm{p}<0.001)$ and at 60 days $(12 \%$ vs $34 \%, \mathrm{p}<0.001)$ even in those cases with macrolide-resistant pathogens. Recently, Rodrigo et al. [37], studied the benefits of beta-lactams/macrolide combination therapy over single beta-lactams therapy for the treatment of immunocompetent adults hospitalized with CAP. They reported that the 30 days inpatient death rate was lower in the combination therapy than in the single therapy group ( $23 \%$ vs $26.8 \%$; OR 0.81 , $95 \%$ CI 0.72 to $0.93, p=0.001$ ). In bacteremic pneumonia Metersky et al. [11], reported that the use of macrolides was associated with lower in-hospital mortality, 30-day mortality and readmission within 30 days of discharge.

Interestingly, the gain in survival and improved outcomes with regard to dual antibiotic therapy, mainly in severe and bacteremic pneumococcal CAP, is associated principally with treatments that include macrolides [38] instead of beta-lactams plus fluoroquinolones [39]. This 
is probably due to the immunomodulatory effect of macrolides.

In vitro and in vivo studies have shown that, due to their immunomodulatory effects, macrolides decrease inflammatory response, independently of antibacterial activity, through different pathways: inhibition of NF-k $\beta$ along with reduction of proinflammatory cytokines production; an inhibitory effect on the release of inflammatory cells such as polymorphonuclear cells; and an effect on structural cells of the respiratory tract that improves mucociliary clearance and increases the expression of molecules tight junctions or $\beta$-defensin [24]. In addition, macrolide antibiotics have an effect on microorganisms because they may inhibit virulence factors production, biofilm formation and protein synthesis [24]. Moreover, a macrolide combination therapy could provide better coverage for atypical microorganisms.

Some other important factors can influence the inflammatory response in CAP patients, such as corticosteroids treatment and comorbidity as COPD [40]. In our study, we corroborated our results with lower IL-6 in BAL and IL-8 and IL-10 in blood after excluding patients treated with corticosteroids.

Our findings are consistent with previous studies as they show an improvement of clinical outcomes (faster clinical stability and shorter LOS) and a reduction of cytokine levels in CAP patients with acute lung infection who are treated with macrolide containing regimens. These findings could be related to decreased levels of the pro-inflammatory cytokines IL- $6, \mathrm{IL}-8$ and TNF- $\alpha$, and support the value of macrolides for their beneficial effect over immunomodulatory properties.

The present study has some limitations that should be considered.

First, the absence of BAL fluid samples in the CAP control group as, for ethical reasons, we did not perform a bronchoscopy in patients with adequate response. There are few human studies that report compartmental inflammatory response in patients with CAP, probably because of the ethical difficulties of performing BAL in non-severe CAP. The current guidelines for management of CAP do not recommend invasive studies when clinical response is appropriate. Second, for our study we enrolled patients after they had received antibiotic treatment. We therefore did not have cytokine levels for those patients prior to initiating their treatment. Third, to improve the robustness of our results, we could ideally have been controlled for patient age and pneumonia severity. However, our study draws upon a single-hospital cohort, and hence upon a limited number of patients. This, and the aforementioned difficulties in collecting data from NCAP patients, including the BAL procedure, prevented our being able to conduct a multivariate analysis for this study.

\section{Conclusions}

Our study shows that after 72 hours of antibiotic therapy, NCAP patients treated with macrolide containing regimens have lower cytokine levels in both compartments (systemic and pulmonary) than those treated with non-macrolide regimens. This supports the immunomodulatory effect of macrolides on cytokine profiles during the course of treatment. That effect seems to contribute to faster resolution and earlier clinical stability. Further randomized trials are needed to confirm the benefit of macrolide therapy in NCAP patients.

\section{Abbreviations}

BAL: Bronchoalveolar lavage; CAP: Community-acquired pneumonia; COPD: Chronic obstructive pulmonary disease; IL: Interleukin; LOS: Length of hospital stay; NCAP: Non-responders CAP; TNF-a: Tumour necrosis factor-a.

\section{Competing interests}

The authors declare that they have no competing interests.

\section{Authors' contributions}

RM designed the study, analyzed, discussed and interpreted the data; she also participated in the revision of the manuscript. MJL participated in study design, statistical analysis and interpretation of data and wrote the manuscript. IM performed the determinations of cytokines, statistical analysis and interpretation of data and contributed to writing the manuscript. BS, EC, $J$ C, participated in study design, interpretation of data and revision of the manuscript. JMM, RM and AG performed the microbiological analysis, participated in the statistical analysis and interpretation of data and revision of the manuscript. All the authors analyzed and discussed the data. All authors read and approved the final manuscript.

\section{Acknowledgements}

This work was supported by the Ciber of respiratory illnesses (CIBERES), an initiative from the Carlos III Health Institute (grant number PI 08/0727), the Bancaja-Fundación para la Investigación Hospital La Fe, Conselleria de Sanidad de la Comunidad Valenciana (grants numbers AP-018/06 and SAF2009-08913) and the public funding from the Fondo de Investigaciones Sanitarias (Instituto de Salud Carlos III, grant number CA10/01027).

\section{Author details}

${ }^{1}$ Pneumology Department, Instituto de Investigación Sanitaria del Hospital Universitario y Politécnico La Fe (IIS La Fe), Valencia, Spain. ${ }^{2}$ Instituto de Investigación Sanitaria del Hospital Universitario y Politécnico La Fe (IIS La Fe), Valencia, Spain. ${ }^{3}$ Pharmacology Department, University of Medicine, Universidad de Valencia, Valencia, Spain. ${ }^{4}$ Medicine-Doctoral Programme Department, Barcelona Autonomous University, Barcelona, Spain. ${ }^{5}$ Research contract IIS La Fe-Fundación Bancaja, Valencia, Spain. ${ }^{6}$ Microbiology Department, Hospital Universitario y Politécnico La Fe, Valencia, Spain. ${ }^{7} \mathrm{CIBER}$ de Enfermedades Respiratorias (CibeRes), Valencia, Spain. ${ }^{8}$ Servicio de Neumología, Hospital Universitario y Politécnico La Fe, Avda Bulevar Sur, 46026 Valencia, Spain.

Received: 11 August 2014 Accepted: 5 January 2015

Published online: 07 February 2015

\section{References}

1. Arancibia F, Ewig S, Martinez JA, Ruiz M, Bauer T, Marcos MA, et al. Antimicrobial treatment failures in patients with community-acquired pneumonia: causes and prognostic implications. Am J Respir Crit Care Med. 2000;162:154-60

2. Ioanas M, Ferrer M, Cavalcanti M, Ferrer R, Ewig S, Filella X, et al. Causes and predictors of non-response to treatment of intensive care unit-acquired pneumonia. Crit Care Med. 2004;32:938-45.

3. Menéndez R, Cavalcanti M, Reyes S, Mensa J, Martinez R, Marcos MA, et al. Markers of treatment failure in hospitalised community-acquired pneumonia. Thorax. 2008;63:447-52 
4. Kellum JA, Kong L, Fink MP, Weissfeld LA, Yealy DM, Pinsky MR, et al. Understanding the inflammatory cytokine response in pneumonia and sepsis. Results of the Genetic and Inflammatory Markers of Sepsis (GenIMS) Study. Arch Intern Med. 2007;167:1655-63.

5. Culic O, Erakovic V, Parnham MJ. Anti-inflammatory effects of macrolide antibiotics. Eur J Pharmacol. 2001;429:209-29.

6. Wales D, Woodhead M. The anti-inflammatory effects of macrolides. Thorax. 1999;54 Suppl 2:58-62.

7. Steel HC, Theron AJ, Cockeran R, Anderson R, Feldman C. Pathogen- and host-directed anti-inflammatory activities of macrolide antibiotics. Mediators Inflamm. 2012;2012:584262. doi:10.1155/2012/584262.

8. Martin-Loeches I, Lisboa T, Rodriguez A, Putensen C, Annane D, GarnachoMontero J, et al. Combination antibiotic therapy with macrolides improves survival in intubated patients with community-acquired pneumonia. Intensive Care Med. 2010;36:612-20.

9. Parnham MJ. Immunomodulatory effects of antimicrobials in the therapy of respiratory tract infections. Curr Opin Infect Dis. 2005;18:125-31.

10. Restrepo MI, Mortensen EM, Waterer GW, Wunderink RG, Coalson JJ, Anzueto A. Impact of macrolide therapy on mortality for patients with severe sepsis due to pneumonia. Eur Respir J. 2009;33:153-9.

11. Metersky ML, Ma A, Houck PM, Bratzler DW. Antibiotics for bacteremic pneumonia: improve outcomes with macrolides but not fluorquinolones. Chest. 2007;131:466-73.

12. Baddour LM, Yu VL, Klugman KP, Feldman C, Ortqvist A, Rello J, et al. International Pneumococcal study group. Combination antibiotic therapy lowers mortality among severily ill patients with pneumococcal pneumonia. Am J Respir Crit Care Med. 2004;170:440-4.

13. Dwyer R, Ortqvist A, Aufwerber E, Henriques Normark B, Marrie TJ, Mufson MA, et al. Addition of a macrolide to a $\beta$-lactam in bacteremic pneumococcal pneumonia. Eur J Clin Microbiol Infect Dis. 2006;25:518-21.

14. Aspa J, Rajas O, Rodriguez DeCastro F, Huertas MC, Borderías L, Cabello FJ, et al. Pneumococcal Pneumonia in Spain study group. Impact of initial antibiotic choice on mortality from pneumococcal pneumonia. Eur Respir J. 2006;27:1010-9.

15. Halm EA, Fine MJ, Marrie TJ, Coley CM, Kapoor WN, Obrosky DS, et al. Time to clinical stability in patients hospitalized with community-acquired pneumonia. JAMA. 1998;279:1452-7.

16. Menéndez R, Torres A, Rodríguez de Castro F, Zalacaín R, Aspa J, Martín Villasclaras JJ, et al. Reaching stability in community-acquired pneumonia: the effects of the severity of disease, treatment, and the characteristics of patients. Clin Infect Dis. 2004;39:1783-90.

17. Fine MJ, Auble TE, Yealy DM, Hanusa BH, Weissfeld LA, Singer DE, et al. A prediction rule to identify low-risk patients with community-acquired pneumonia. N Engl J Med. 1997;336:243-50

18. Alfageme I, Aspa J, Bello S, Blanquer J, Blanquer R, Borderías L, et al. Guidelines for the diagnosis and management of community-acquired pneumonia. Spanish Society of Pneumology and Thoracic Surgery (SEPAR) Arch Bronconeumol. 2005;41:272-89.

19. Technical recommendations and guidelines for bronchoalveolar lavage. Report of the European Society of Pneumology Task Group. Eur Respir J. 1989:2:561-585

20. Montón C, Torres A, El-Ebiary M, Filella X, Xaubet A, de la Bellacasa JP. Cytokine expression in severe pneumonia: a bronchoalveolar lavage study. Crit Care Med. 1999;27:1745-53.

21. Paats MS, Bergen IM, Hanselaar WE, van Zoelen ECG, Hoogsteden HC, Hendriks RW, et al. Local and systemic cytokine profiles in nonsevere and severe community-acquired pneumonia. Eur Respir J. 2013:41:1378-85.

22. Moret I, Lorenzo MJ, Sarria B, Cases E, Morcillo E, Perpiñá M, et al. Increased lung neutrophil apoptosis and inflammation resolution in nonresponding pneumonia. Eur Respir J. 2011:38:1158-64.

23. Corrales-Medina VF, Musher DM. Immunomodulatory agents in the treatment of community-acquired pneumonia: a systematic review. J Infect. 2011;63:187-99.

24. Meijvis SC, van de Garde EM, Rijkers GT, Bos WJ. Treatment with antiinflammatory drugs in community-adquired pneumonia. J Intern Med. 2012;272:25-35.

25. Kovaleva A, Remmelts HH, Rijkers GT, Hoepelman Al, Biesma DH, Oosterheert JJ. Immunomodulatory effects of macrolides during community-acquired pneumonia: a literature review. J Antimicrob Chemother. 2012;67:530-40.

26. Steel HC, Cockeran R, Anderson R, Feldman C. Overview of communityacquired pneumonia and the role of inflammatory mechanisms in the immunopathogenesis of severe pneumococcal disease. Mediators Inflamm. 2013:2013:490346. doi:10.1155/2013/490346. Epub 2013 Dec 25.

27. Demartini G, Esposti D, Marthyn P, Lapidari A, Fraschini F, Scaglione F. Effect of multiple doses of clarithromycin and amoxicillin on IL-6, IFN gamma and IL-10 plasma levels in patients with community acquired pneumonia. J Chemother. 2004;16:82-5.

28. Spyridaki A, Raftogiannis M, Antonopoulou A, Tsaganos T, Routsi C, Baziaka $F$, et al. Effect of clarithromycin in inflammatory markers of patients with ventilator-associated pneumonia and sepsis caused by gram-negative bacteria: results from a randomized clinical study. Antimicrob Agents Chemother. 2012;56:3819-25.

29. Nakamura S, Yanagihara K, Araki N, Yamada K, Morinaga Y, Izumikawa K, et al. Efficacy of clarithromycin against experimentally induced pneumonia caused by clarithromycin-resistant Haemophilus influenzae in mice. Antimicrob Agents Chemother. 2010:54:757-62.

30. Sanz MJ, Nabah YN, Cerdá-Nicolás M, O'Connor JE, Issekutz AC, Cortijo J, et al. Erythromycin exerts in vivo anti-inflammatory activity downregulating cell adhesion molecule expression. Br J Pharmacol. 2005;144:190-201.

31. Beigelman A, Mikols CL, Gunsten SP, Cannon CL, Brody SL, Walter MJ. Azithromycin attenuates airway inflammation in a mouse model of viral bronchiolitis. Respir Res. 2010;11:90.

32. Amado-Rodríguez L, González-López A, López-Alonso I, Aguirre A, Astudillo

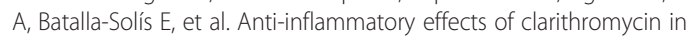
ventilator-induced lung injury. Respir Res. 2013;14:52.

33. Anderson R, Steel HC, Cockeran R, von Gottberg A, de Gouveia L, Klugman $\mathrm{KP}$, et al. Clarithromycin alone and in combination with ceftriaxone inhibits the production of pneumolysin by both macrolide-susceptible and macrolide-resistant strains of Streptococcus pneumoniae. J Antimicrob Chemother. 2007:59:224-9.

34. Martínez JA, Horcajada JP, Almela M, Marco F, Soriano A, García E, et al. Addition of a macrolide to a beta-lactam-based empirical antibiotic regimen is associated with lower in-hospital mortality for patients with bacteremic pneumococcal pneumonia. Clin Infect Dis. 2003;36:389-95.

35. Weiss K, Low DE, Cortes L, Beaupre A, Gauthier R, Gregoire P, et al. Clinical characteristics at initial presentation and impact of dual therapy on the outcome of bacteremic Streptococcus pneumoniae pneumonia in adults. Can Respir J. 2004;11:589-93.

36. Mufson MA, Stanek RJ. Revisiting combination antibiotic therapy for community-acquired invasive streptococcus pneumoniae pneumonia. Clin Infect Dis. 2006;42:304-6.

37. Rodrigo C, Mckeever TM, Woodhead M, Lim WS, On behalf of the British Thoracic Society. Single versus combination antibiotic therapy in adults hospitalized with community-acquired pneumonia. Thorax. 2013;68:493-5.

38. Waterer GW, Rello J, Wunderink RG. Management of community-acquired pneumonia in adults. Am J Respir Crit Care Med. 2011;183:157-64.

39. Mortensen EM, Restrepo MI, Anzueto A, Pugh J. The impact of empiric antimicrobial therapy with $\beta$-lactam and fluoroquinolone on mortality for patients hospitalized with severe pneumonia. Crit Care Med. 2005:10:R8

40. Crisafulli E, Menéndez R, Huerta A, Martinez R, Montull B, Clini E, et al. Systemic inflammatory pattern of patients with community-acquired pneumonia with and without COPD. Chest. 2013;143:1009-17.

\section{Submit your next manuscript to BioMed Central and take full advantage of:}

- Convenient online submission

- Thorough peer review

- No space constraints or color figure charges

- Immediate publication on acceptance

- Inclusion in PubMed, CAS, Scopus and Google Scholar

- Research which is freely available for redistribution 